\title{
Subthreshold antiproton production in nucleus-nucleus collisions
}

\author{
G. Q. Li, C. M. Ko, X. S. Fang, and Y. M. Zheng* \\ Cyclotron Institute and Physics Department, Texas A $\mathcal{B} M$ University, College Station, Texas 77843
}

(Received 17 September 1993; revised manuscript received 3 November 1993)

\begin{abstract}
Antiproton production in nucleus-nucleus collisions at energies below the threshold for its production from the nucleon-nucleon interaction in free space is studied in the relativistic Vlasov-UehlingUhlenbeck model. The antiproton self-energies in the medium are obtained in the mean-field approximation from the $G$-parity transformation of the nucleon self-energies. Due to the decrease of antiproton mass in the medium as a result of the attractive scalar mean field, there is an enhanced production of primordial antiprotons which are, however, mostly annihilated by nucleons as they propagate through the matter. The calculated final antiproton momentum spectrum is seen to agree reasonably with the experimental data.
\end{abstract}

PACS number(s): 25.75. $+\mathrm{r}, 24.10 . \mathrm{Jv}$

\section{INTRODUCTION}

Particle production in nucleus-nucleus collisions at energies that are below the threshold for its production from the nucleon-nucleon interaction in free space offers the possibility to study the properties of hadronic matter at high densities [1-5]. Experiments have already been carried out to study the production of kaons $[6,7]$, antikaons [8], and antiprotons [8,9] from heavy-ion collisions at subthreshold energies. With a threshold at 5.6 $\mathrm{GeV}$, antiproton production in nucleus-nucleus collisions at energies of a few $\mathrm{GeV} /$ nucleon is clearly the most extreme subthreshold process in particle production. The first observation of subthreshold antiproton production in proton-nucleus collisions dates back to the 1950s [10] and 1960s $[11,12]$. The experiments at the BEVALAC [8] and the JINR [9] in the 1980s provided the first evidence of subthreshold antiproton production in nucleus-nucleus collisions. Recently, new measurements of subthreshold antiproton production have been carried out at the KEK [13] and at the GSI [14].

There have been attempts to explain theoretically subthreshold antiproton production using, e.g., the fireball model $[15,16]$, the first-chance nucleon-nucleon collision model [17], the quasicoherent multiparticle collision model [18], and the dynamical models such as the VlasovUehling-Uhlenbeck (VUU) model [19] and the quantum molecular dynamics [20]. In the fireball model, one assumes that the system has reached thermal equilibrium and antiprotons are produced from nucleon resonances [15] or meson-meson interactions (e.g., $\rho \rho \rightarrow p \bar{p}$ ) [16]. In these calculations $[15,16]$, however, no detailed comparison with experimental antiproton momentum spectra was made.

The first-chance nucleon-nucleon collision model was used by Shor et al. [17] to study subthreshold antiproton production in proton-nucleus and nucleus-nucleus colli-

\footnotetext{
*Permanent address: Institute of Atomic Energy, Beijing 102413, China.
}

sions. In this model, they included the internal nuclear momentum distribution extracted from the data on quasielastic electron scattering and backward proton production. Using a proper transition matrix element for $N N \rightarrow N N p \bar{p}$, Shor et al. [17] were able to reproduce antiproton spectra in proton-nucleus collisions, but underestimated (with the same transition matrix element) the data in nucleus-nucleus collisions by more than three orders of magnitude. The underestimation will be even larger if one treats antiproton annihilation explicitly which is more significant in nucleus-nucleus collisions than in proton-nucleus collisions.

The first investigation of subthreshold antiproton production in the framework of transport models was done by Batko et al. [19]. In this calculation, the $\Delta(1232)$ degree of freedom was taken into account and was found to play a significant role in subthreshold antiproton production. Neglecting the antiproton mean field and assuming that $90 \%$ of the primordial antiprotons are annihilated, Batko et al. [19] achieved a reasonable description of antiproton production in proton-nucleus collisions, but still underestimated the data in nucleus-nucleus collisions by about a factor of 5 . A similar calculation was carried out by Huang et al. [20] in quantum molecular dynamics (QMD). Again the antiproton production data in nucleus-nucleus collisions were underestimated by about the same order of magnitude as in Ref. [19]. In both calculations $[19,20]$, antiproton annihilation was treated schematically and the propagation as well as the elastic rescattering of antiprotons in the medium were neglected.

The underestimation of the antiproton yield in nucleusnucleus collisions has led to the suggestion that the quasiparticle properties of baryons (especially the reduction of baryon masses) in a dense medium might be important $[21,22]$. Of equal importance to a proper understanding of the experimental data is the consistent treatment of antiproton production, propagation, rescattering, and most importantly, annihilation in the hadronic matter. In this paper, we shall investigate in detail antiproton production in nucleus-nucleus collisions in the framework of the relativistic Vlasov-Uehling-Uhlenbeck (RVUU) model, which is generalized to include the an- 
tiproton degree of freedom. In this way we can include the medium effects on antiprotons and treat consistently their production, propagation, rescattering as well as annihilation.

This paper is organized as follows. In Sec. II, we briefly review the RVUU model. The extension of RVUU to antiproton production and propagation in matter is outlined in Sec. III. The results and discussions are presented in Sec. IV. Finally, a summary is given in Sec. V.

\section{RELATIVISTIC}

\section{VLASOV-UEHLING-UHLENBECK MODEL}

Our starting point is the following nonlinear $\sigma-\omega$ model $[23,24]$ :

$$
\begin{aligned}
\mathcal{L}= & \bar{\psi}\left[i \gamma_{\mu} \partial^{\mu}-m-g_{\sigma} \sigma-g_{\omega} \gamma_{\mu} \omega^{\mu}\right] \psi+\frac{1}{2}\left(\partial^{\mu} \sigma\right)^{2} \\
& -\frac{1}{2} m_{\sigma}^{2} \sigma^{2}-\frac{1}{3} b \sigma^{3}-\frac{1}{4} c \sigma^{4}-\frac{1}{4}\left(\partial_{\mu} \omega^{\nu}-\partial_{\nu} \omega^{\mu}\right)^{2} \\
& +\frac{1}{2} m_{\omega}^{2} \omega^{\mu 2}
\end{aligned}
$$

where $\psi$ is the nucleon field with mass $m$, while $\sigma$ and $\omega$ are the scalar and vector meson fields with masses $m_{\sigma}$ and $m_{\omega}$, respectively. The coupling constants of the nucleon to the sigma and omega mesons are denoted, respectively, by $g_{\sigma}$ and $g_{\omega}$. The $\sigma^{3}$ and $\sigma^{4}$ terms represent the scalar meson self-interaction.

For a quasihomogeneous and weakly interacting nuclear system, one can derive a transport equation (RVUU equation) for the phase space distribution function $f\left(x, \mathbf{p}^{*}\right)$ of nucleons $[22,25-27]$, i.e.,

$$
\begin{aligned}
\frac{1}{p_{0}^{*}}\left\{\left[\partial_{x}^{\mu}-\left(\partial_{x}^{\mu} \Sigma_{v}^{\nu}-\partial_{x}^{\nu} \Sigma_{v}^{\mu}\right) \partial_{\nu}^{p^{*}}\right] p_{\mu}^{*}\right. & \\
& \left.+m^{*}\left(\partial_{x}^{\mu} m^{*}\right) \partial_{\mu}^{p^{*}}\right\} f\left(x, \mathbf{p}^{*}\right)=I_{c}
\end{aligned}
$$

where $I_{c}$ is the collision term. In the above, the nucleon effective mass $m^{*}$ and the kinetic momentum $p_{\mu}^{*}$ are defined by

$$
m^{*}=m-\Sigma_{s}, \quad p_{\mu}^{*}=p_{\mu}-\Sigma_{v \mu}
$$

where the nucleon scalar $\left(\Sigma_{s}\right)$ and vector $\left(\Sigma_{v \mu}\right)$ selfenergies are given by

$$
\Sigma_{s}=g_{\sigma}\langle\sigma\rangle, \quad \Sigma_{v \mu}=g_{\omega}\left\langle\omega_{\mu}\right\rangle
$$

In the mean-field approximation, the expectation values of the scalar and vector fields in nuclear medium are related to the nuclear scalar density $\rho_{s}$ and current den- sity $\rho_{\mu}$, respectively,

$$
\begin{gathered}
m_{\sigma}^{2}\langle\sigma\rangle+b\langle\sigma\rangle^{2}+c\langle\sigma\rangle^{3} \approx g_{\sigma} \rho_{s}, \\
\left\langle\omega_{\mu}\right\rangle=\left(g_{\omega} / m_{\omega}^{2}\right) \rho_{\mu} .
\end{gathered}
$$

In terms of the phase space distribution function, the scalar and current densities in the local-density approximation can be expressed, respectively, as

$$
\rho_{s}=\int \frac{d^{3} \mathbf{p}^{*}}{(2 \pi)^{3}} f\left(x, \mathbf{p}^{*}\right) m^{*} / E^{*}
$$

and

$$
\rho_{\mu}=\int \frac{d^{3} \mathbf{p}^{*}}{(2 \pi)^{2}} f\left(x, \mathbf{p}^{*}\right) p_{\mu}^{*} / E^{*}
$$

where $E^{*}=\left(m^{* 2}+\mathbf{p}^{* 2}\right)^{1 / 2}$.

As usual, we use $m_{\sigma}=550 \mathrm{MeV}$ and $m_{\omega}=783 \mathrm{MeV}$. The coupling constants and the sigma-meson self-interaction strength are determined by fitting the nuclear matter saturation properties, i.e., a saturation density $\rho_{0}=0.16$ $\mathrm{fm}^{-3}$ and an energy per nucleon $(\mathcal{E} / A)_{\rho_{0}}=-15.96 \mathrm{MeV}$. Furthermore, we fix the nucleon effective mass at $\rho_{0}$ to be $m^{*}=0.83 m$. The nuclear matter incompressibility $K$ at $\rho_{0}$ is chosen to be $200 \mathrm{MeV}$ for a soft equation of state and $380 \mathrm{MeV}$ for a stiff equation of state, respectively. In this way, we have determined two sets of parameters which are shown in Table I. These parameters are slightly different from those given in Ref. [25] where the saturation density was assumed to be $0.145 \mathrm{fm}^{-3}$.

In Fig. 1 we show the density dependence of the energy per nucleon $\mathcal{E} / A$ for the two sets of parameters. The solid and dashed curves correspond to nuclear incompressibility of $200 \mathrm{MeV}$ and $380 \mathrm{MeV}$, respectively. We note that the difference between the two equations of state is smaller than that in the nonrelativistic Skyrme parametrizations $[4,5]$. In Fig. 2 the density dependence of the nucleon effective mass $m^{*}$ is shown by the solid curve for the soft equation of state and the dashed curve for the stiff equation of state. In both cases the nucleon effective mass decreases with increasing density, and the reduction is larger for the soft equation of state than for the stiff one. Although the effective masses at the saturation density for the two sets of parameters are taken to be the same, they differ at high densities.

The RVUU equation is solved by the method of pseudoparticle [28]. In this method, each nucleon is replaced by a collection of test particles, and the one-body phase space distribution function is given by the distribution of these particles in phase space. To solve the Vlasov equation, i.e., the left-hand side of Eq. (2), is then equivalent to the solution of the following classical equations of mo-

TABLE I. Parameters of the nonlinear $\sigma-\omega$ model corresponding to the soft and stiff equations of state and nuclear matter properties at the saturation density $\rho_{0}=0.16 \mathrm{fm}^{-3}$.

\begin{tabular}{lccccccc}
\hline \hline & $C_{\sigma}=\left(g_{\sigma} / m_{\sigma}\right) m$ & $C_{\omega}=\left(g_{\omega} / m_{\omega}\right) m$ & $B=b /\left(g_{\sigma}^{3} m\right)$ & $C=c / g_{\sigma}^{4}$ & $\mathcal{E} / A(\mathrm{MeV})$ & $m^{*} / m$ & $K(\mathrm{MeV})$ \\
\hline soft & 13.95 & 8.498 & 0.0199 & -0.00296 & -15.96 & 0.83 & 200.0 \\
stiff & 11.27 & 8.498 & -0.0283 & 0.1859 & -15.96 & 0.83 & 380.0 \\
\hline \hline
\end{tabular}




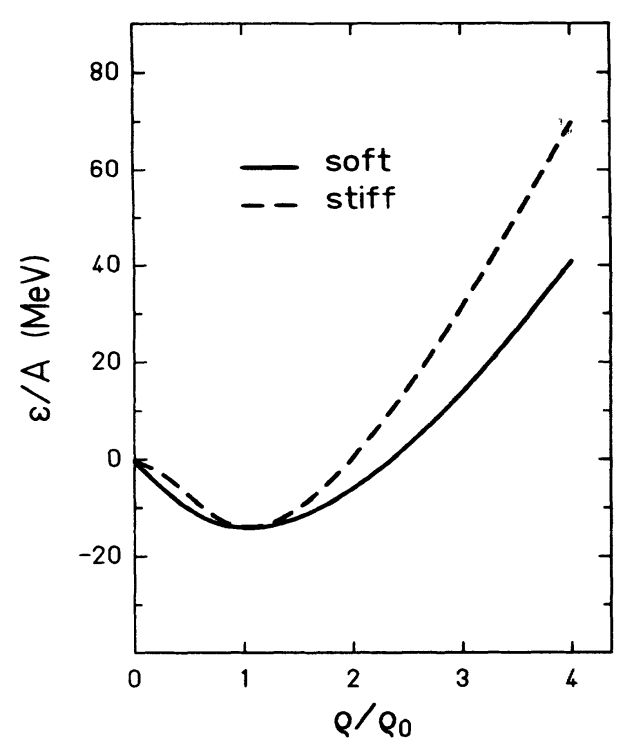

FIG. 1. Soft (solid curve) and stiff (dashed curve) equations of state of nuclear matter corresponding to the parameters listed in Table I.

tion for all test particles:

$$
\begin{aligned}
& \frac{d \mathbf{x}}{d t}=\mathbf{p}^{*} / E^{*}, \\
& \frac{d \mathbf{p}}{d t}=-\nabla_{\mathbf{x}}\left[E^{*}+\left(g_{\omega} / m_{\omega}\right)^{2} \rho\right],
\end{aligned}
$$

where $\rho$ is nuclear matter density.

The in-medium two-body collisions are treated in the same way as in cascade model [29] and Vlasov-UehlingUhlenbeck (VUU) model [30]. The following elastic and inelastic processes are included:

$$
N N \rightarrow N N, N \Delta \rightarrow N \Delta, \Delta \Delta \rightarrow \Delta \Delta,
$$

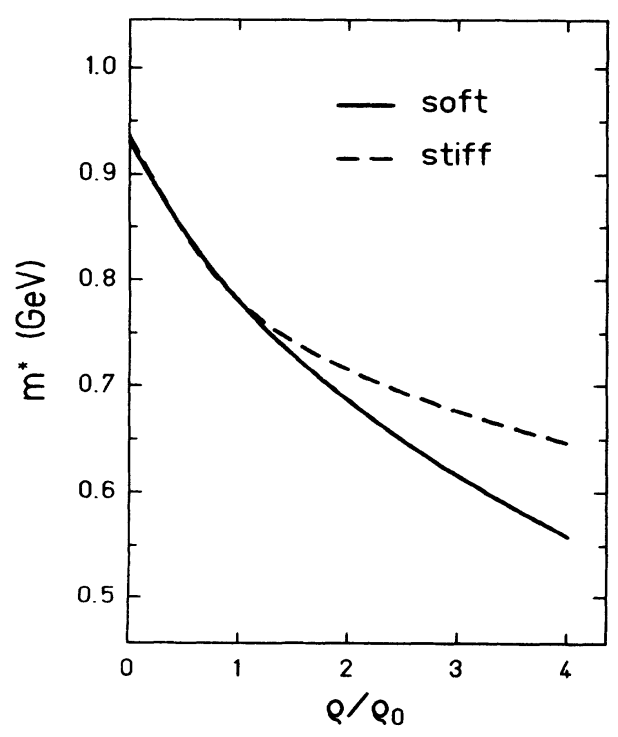

FIG. 2. The density dependence of the nucleon effective mass corresponding to the soft (solid curve) and stiff (dashed curve) equations of state.

$$
N N \rightarrow N \Delta, N \Delta \rightarrow N N .
$$

For $N N \rightarrow N N$ and $N N \rightarrow N \Delta$, the isospin-averaged nucleon-nucleon cross sections in free space $[29,30]$ are used. The cross sections for $N \Delta \rightarrow N \Delta$ and $\Delta \Delta \rightarrow \Delta \Delta$ are assumed to be the same as that for $N N \rightarrow N N$ at the same center-of-mass energy. The cross section for $N \Delta \rightarrow$ $N N$ is determined from the detailed balance relation [31]. Deltas are also propagated according to Eqs. (9) and (10) with their self-energies taken to be similar to the nucleon self-energies.

Finally, the pion degree of freedom is introduced via delta decay, i.e., $\Delta \rightarrow \pi N$. The inverse process $\pi N \rightarrow \Delta$ is also included to take into account the pion absorption. Pions are assumed to propagate as free particles in the hadronic matter. As we shall show later, antiprotons are produced mainly from $N \Delta$ and $\Delta \Delta$ interactions. To calculate the antiproton yield in nucleus-nucleus collisions requires thus the proper treatment of both delta formations and decays.

Due to the strong annihilation effect in antiprotonnucleus scatterings, the mean-field potential of an antiproton inside a nucleus is not well known empirically. However, in the mean-field approximation the antinucleon self-energies can be obtained from the $G$-parity transformation, or charge conjugation, of the nucleon self-energies [32]. Since the scalar field remains unchanged under the $G$-parity transformation, the antiproton mass in the medium is similar to the nucleon mass and thus decreases with increasing density (see Fig. 2). This reduces the threshold for antiproton production in the nuclear medium and leads to an enhanced production of the primordial antiprotons in nucleus-nucleus collisions. The vector potential acting on the antiproton has, however, an opposite sign from that for nucleons and is thus negative. We show in Fig. 3 the density dependence of the antiproton scalar and vector (the timelike

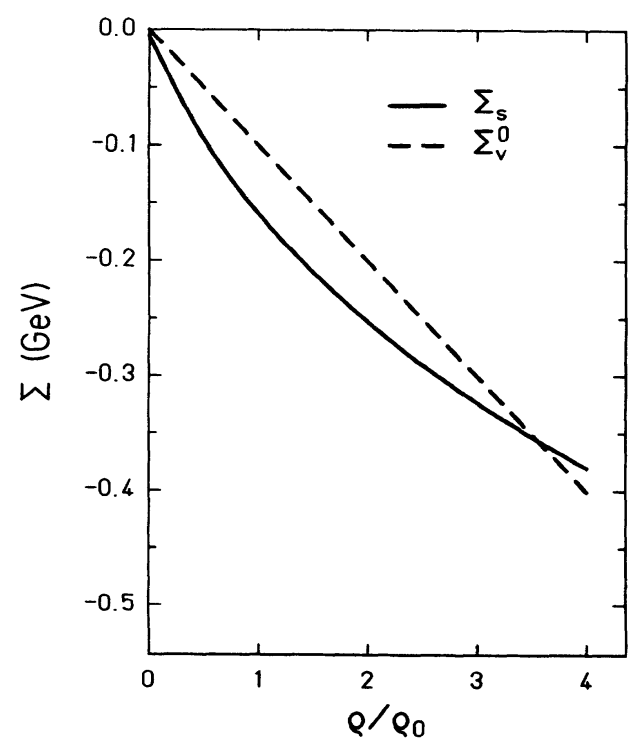

FIG. 3. The antiproton scalar (solid curves) and vector (dashed curves) potentials corresponding to the soft equations of state. 
component) potentials corresponding to the soft equation of state. For the stiff equation of state, the vector potential is the same as for the soft equation of state, while the scalar potential has a smaller magnitude (see Fig. 2).

The assumption that the antiproton potential is related to the nucleon potential simply by the $G$-parity transformation has not been proved. Beyond the meanfield approximation, it is likely that this is not valid as higher-order contributions may transform differently un$\operatorname{der} G$ parity. However, most experimental results from heavy-ion collisions at high energies can be successfully described by the transport model with mean-field potentials and two-body collisions. Without empirical information on the antiproton potential, it is reasonable to first treat the antiproton potential in the mean-field approximation. In this way, both nucleons and antinucleons are treated on the same footing in the RVUU model. The validity of this assumption can be tested by comparing the theoretical results with the experimental data.

\section{ANTIPROTON PRODUCTION AND PROPAGATION}

Antiprotons are assumed to be produced mainly from the baryon-baryon interaction, i.e., $B B \rightarrow N N p \bar{p}$, where $B$ denotes either a nucleon or a delta. Because of the small probability of antiproton production in baryonbaryon interactions, antiproton production in nucleusnucleus collisions at subthreshold energies is treated perturbatively, and so the collision dynamics is not affected by the presence of produced antiprotons.

The antiproton momentum spectrum in the protonproton interaction in free space has been parametrized as $[10,11,18,33]$

$$
\frac{E d^{2} \sigma_{p p}^{\bar{p}}}{p^{2} d p d \Omega}=\sigma_{p p}^{\bar{p}}(\sqrt{s}) E\left(p_{\max }^{2}-p^{2}\right)^{2} \frac{105}{32 \pi p_{\max }^{7}}
$$

where $p$ and $E$ are the antiproton momentum and energy in the proton-proton center-of-mass frame, respectively. The maximum momentum of the antiproton, $p_{\max }$, is related to the available center-of-mass energy $\sqrt{s}$ of the proton pair by

$$
p_{\max }=\left[\frac{1}{4 s}\left(s-16 m^{2}\right)\left(s-4 m^{2}\right)\right]^{1 / 2} .
$$

This parametrization is based on the phase space argument and describes reasonably well experimental antiproton momentum spectrum in proton-proton collisions $[10,11,18]$. The total antiproton production cross section in Eq. (11) is fitted to the available experimental data $[34,35]$, i.e.,

$$
\sigma_{p p}^{\bar{p}}(\sqrt{s})=0.012\left(\sqrt{s}-\sqrt{s_{0}}\right)^{1.846} \mathrm{mb} .
$$

In free space, we have $\sqrt{s_{0}}=4 m$. In Fig. 4, we show a comparison of this parametrization with the available experimental data, where open and solid circles are taken from Ref. [34] and Ref. [35], respectively.

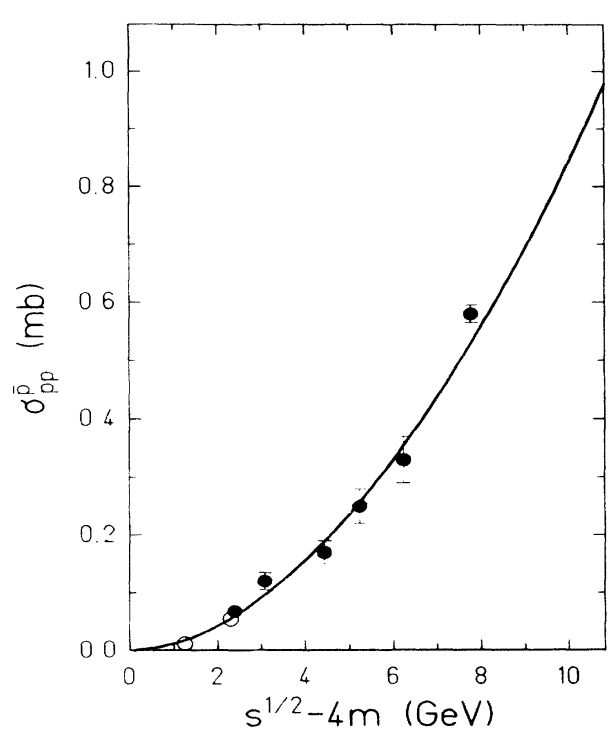

FIG. 4. The parametrization of antiproton production cross section in the proton-proton interaction, as proposed in Ref. [19]. The experimental data are from Ref. [33] (open circles) and Ref. [34] (solid circles).

Medium effects on the antiproton differential spectrum in nucleus-nucleus collisions can be included by evaluating Eqs. (12) and (13) with the in-medium nucleon and antiproton masses. The total center-of-mass energy $\sqrt{s}$ of the colliding pair of baryons is then given by

$$
\sqrt{s^{*}}=\left(\mathbf{p}_{1}^{*}+m_{1}^{*}\right)^{1 / 2}+\left(\mathbf{p}_{2}^{*}+m_{2}^{*}\right)^{1 / 2},
$$

where $m_{i}^{*}$ and $\mathbf{p}_{i}^{*}(i=1,2)$ are, respectively, the effective mass and kinetic momentum of the colliding baryons.

We assume that the antiproton production cross section in all baryon-baryon collisions is the same as that in proton-proton collisions, given by Eqs. (11)-(13), at the same center-of-mass energy. Since most antiprotons are produced in $N \Delta$ and $\Delta \Delta$ interactions (see discussion in Sec. IV), it will be of interest to investigate if this assumption is valid.

We note that, within the mean-field approximation and under the $G$-parity transformation, the vector potential energies are the same in both initial and final states of the reaction $B B \rightarrow N N p \bar{p}$ and thus do not play any role in primordial antiproton production.

In order to have good statistics and to treat properly antiproton rescattering as well as annihilation, we artificially allow $\mathcal{N}_{\bar{p}}$ antiprotons to be produced in each baryon-baryon collision with energy above the antiproton production threshold. The momentum distribution of these antiprotons is determined according to Eq. (11). Eaçh antiproton carries then a production probability which is given by $1 / \mathcal{N}_{\bar{p}}$ time the ratio of the antiproton production cross section to the baryon-baryon total cross section. The propagation of antiprotons in the hadronic matter is then followed by solving the following classical equations of motion:

$$
\frac{d \mathbf{x}_{\bar{p}}}{d t}=\mathbf{p}_{\bar{p}}^{*} / E_{\overline{\mathbf{p}}}^{*}
$$




$$
\frac{d \mathbf{p}_{\bar{p}}}{d t}=-\nabla_{\mathbf{x}}\left[E_{\bar{p}}^{*}-\left(g_{\omega} / m_{\omega}\right)^{2} \rho\right] .
$$

Furthermore, antiprotons can be rescattered or annihilated by baryons when they travel through the matter. This is treated as in the cascade model by using the following parametrizations for the antiproton elastic and annihilation cross sections [36], which are fitted to the available antiproton-proton experimental data [37]:

$$
\begin{aligned}
\sigma_{\text {ela }}^{\bar{p}} & =42.3 / p_{\text {lab }}^{0.54}+4.3 \exp \left[-\left(p_{\text {lab }}-1.5\right)^{2}\right], \\
\sigma_{\mathrm{ann}}^{\bar{p}} & =24 / p_{\text {lab }}^{1.1}+38 / p_{\text {lab }}^{0.5},
\end{aligned}
$$

where $p_{\text {lab }}$ is the momentum of the antiproton in the rest frame of the struck proton. The variation of the elastic and annihilation cross sections with antiproton laboratory momentum is shown in Fig. 5. We assume that antiproton-baryon elastic and annihilation cross sections are the same as the antiproton-proton ones, given by Eqs. (17) and (18), at the same antiproton momentum $p_{\text {lab. }}$. For the range of antiproton momenta involved in this work, other processes, such as the charge exchange one, are unimportant [36].

When the closest distance between an antiproton and a baryon is less than $\sqrt{\left(\sigma_{\text {ela }}+\sigma_{\text {ann }}\right) / \pi}$, a collision occurs, and it can be either an elastic or an annihilation process, depending on the relative magnitudes of $\sigma_{\text {ela }}$ and $\sigma_{\text {ann }}$. If an annihilation process takes place, the antiproton disappears and is no longer propagated. If it is an elastic one, the direction of antiproton momentum after the collision is assumed to be isotropic. In both cases, the baryon momentum is unchanged as we have treated perturbatively antiproton production in nucleus-nucleus collisions.

Finally, the Lorentz-invariant antiproton differential cross section in the nucleus-nucleus collision is obtained by integrating the corresponding differential multiplici-

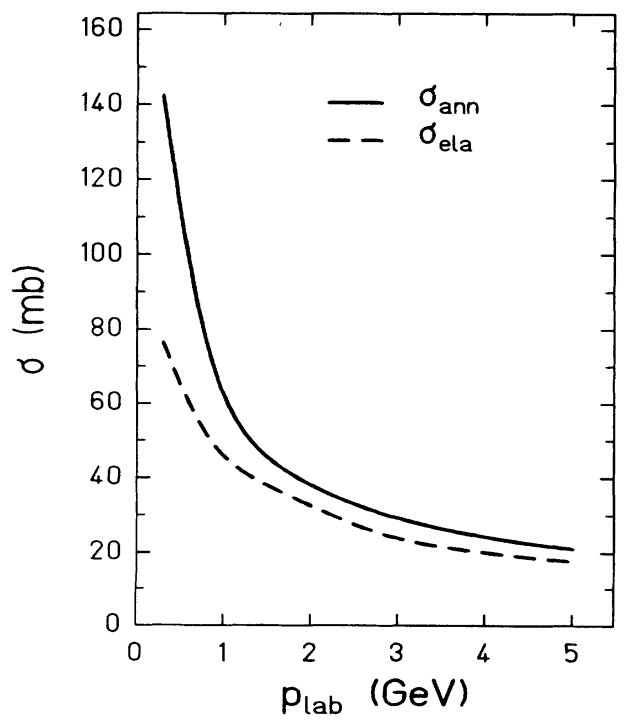

FIG. 5. Parametrizations of antiproton annihilation (solid curve) and elastic (dashed curve) cross sections as proposed in Ref. [36] by fitting the experimental data of Ref. [37]. ties over the impact parameter $b$, i.e.,

$$
\frac{E d^{2} \sigma}{p^{2} d p d \Omega}=2 \pi \int b d b \frac{E d^{2} N(b)}{p^{2} d p d \Omega}
$$

where $E d^{2} N(b) / p^{2} d p d \Omega$ includes contributions from all baryon-baryon collisions with energies above the antiproton production threshold that occur during the nucleusnucleus collision at a given impact parameter $b$.

\section{RESULTS AND DISCUSSIONS}

With the formalism outlined in Secs. II and III, we can calculate the antiproton production cross section in nucleus-nucleus collisions. Before showing the comparison of our theoretical results with the experimental data, we discuss various aspects of our model and their effects on antiproton production in nucleus-nucleus collisions. Unless otherwise stated, results presented in this section are obtained with the RVUU model and the soft equation of state.

We show in Fig. 6 the time evolution of the central baryon density (solid curve), the delta density (dashed curve), and the pion density (dotted curve) in a headon $(b=0 \mathrm{fm}){ }^{28} \mathrm{Si}+{ }^{28} \mathrm{Si}$ collision at $2.1 \mathrm{GeV} /$ nucleon. It is seen that in the early stage of the collision a dense baryon matter with density at about $2.5 \rho_{0}$ is formed and lasts for a few $\mathrm{fm} / c$. About $25 \%$ of the particles in this dense matter are deltas, and so it is not unreasonable to say that a delta matter has been formed in heavy-ion reactions at energies about a few $\mathrm{GeV} /$ nucleon.

In Fig. 7 we show the particle abundance as a function of time for the same collision as in Fig. 6. The solid, dotted, and dashed curves give the nucleon, delta, and pion numbers, respectively. The antiproton number is shown

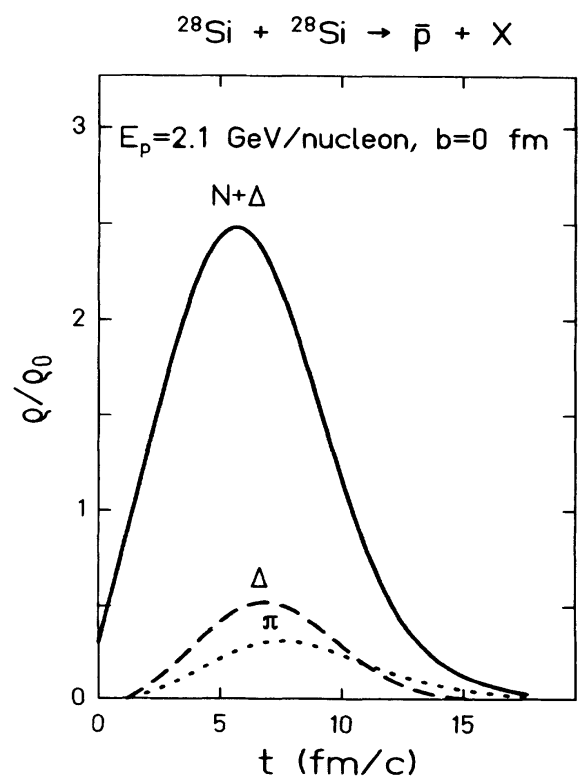

FIG. 6. The time evolution of the central baryon (nucleon + deltas) density (solid curve), the delta density (dashed curve), and the pion density (dotted curve). 


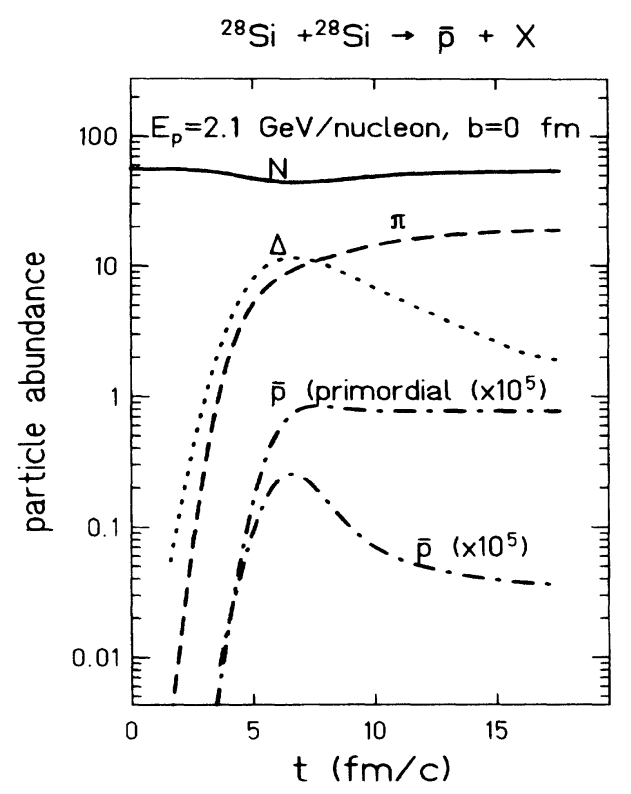

FIG. 7. The time evolution of particle abundance. The two dash-dotted curves correspond to the primordial and final (with antiproton annihilation effect included) antiproton abundance, respectively.

by two dash-dotted curves, one for primordial antiprotons and the other for final antiprotons after taking into account annihilation. The primordial antiproton abundance is about $8 \times 10^{-6}$ but is reduced to about $3.2 \times 10^{-7}$ due to annihilation; i.e., only about $4 \%$ of primordial antiprotons can escape from the dense hadronic matter and be detected. The schematic calculation of Ref. [19], which assumes that the annihilation is $90 \%$, therefore underestimates antiproton annihilation by about a factor of $2-3$. Antiprotons are mainly produced in the early stage of the collision (before $7 \mathrm{fm} / c$ ) when the matter density is high. Including annihilation, the antiproton number reaches its maximum value at about $6 \mathrm{fm} / c$ when the delta abundance is high. It then decreases rapidly and eventually freezes out at about $18 \mathrm{fm} / c$, when the baryon density is about $1 / 10 \rho_{0}$.

The individual contributions from $N N, N \Delta$, and $\Delta \Delta$ interactions to the total primordial antiproton production probability are shown in Fig. 8 . We see that the largest contribution comes from the $N \Delta$ interaction, which accounts for about half the total probability. The $\Delta \Delta$ contribution is about equally important as the $N N$ contribution. The importance of delta resonances in subthreshold particle production has also been observed in subthreshold kaon production from a $\mathrm{Au}+\mathrm{Au}$ collision at $1 \mathrm{GeV} /$ nucleon $[22,38,39]$.

To see the effect of nuclear equation of state on the antiproton production probability, we carry out two calculations for ${ }^{28} \mathrm{Si}+{ }^{28} \mathrm{Si}$ collision at $2.1 \mathrm{GeV} /$ nucleon and $b=0 \mathrm{fm}$, one with a soft equation of state and the other with a stiff equation of state (for their parameters see Table I). We show in Fig. 9 the time evolution of the primordial antiproton production probabilities obtained in the two calculations. It is seen that the an-

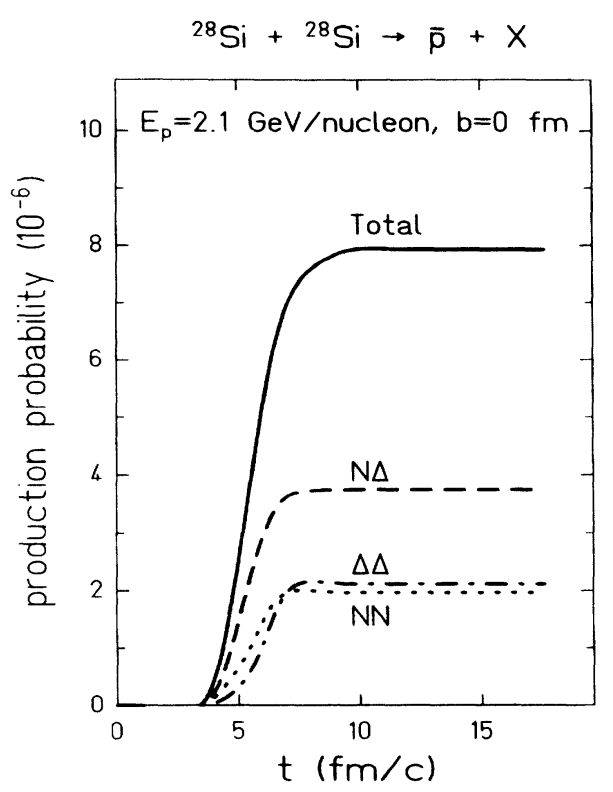

FIG. 8. The contributions of $N N, N \Delta$, and $\Delta \Delta$ interactions to the primordial antiproton production probability.

tiproton production probability with the soft equation of state is about $50 \%$ larger than that with the stiff equation of state. This enhancement in the case of a soft equation of state is partly due to a small incompressibility (thus a small compressional energy; see Fig. 1) and partly due to a small effective mass at high density (thus a lower threshold; see Fig. 2). The final antiproton production probability shows similar dependence on the nuclear equation of state, as the annihilation effect does not depend much on the nuclear equation of state.

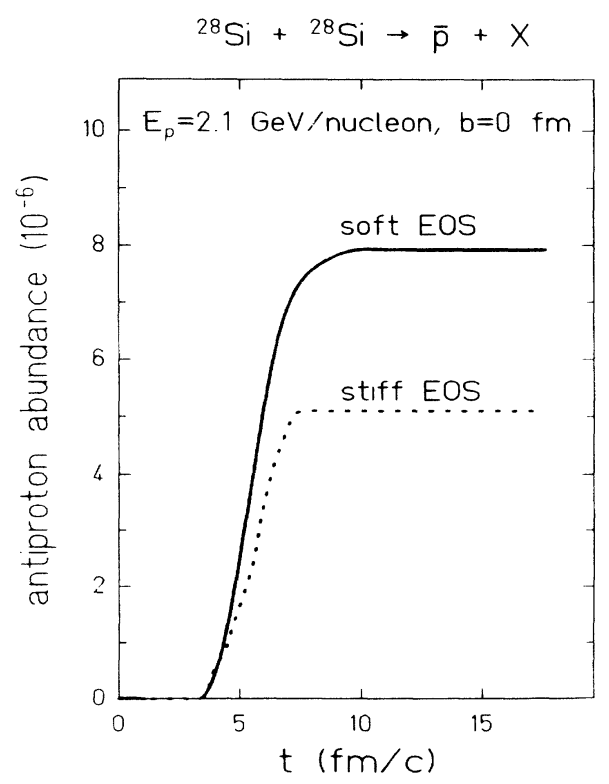

FIG. 9. The primordial antiproton production probability corresponding to the soft (solid curve) and stiff (dashed curve) equation of state. 
The effect due to the nuclear equation of state will be larger if heavier systems are considered. However, given the uncertainties in the in-medium elementary antiproton production cross section and antiproton annihilation cross section, it is doubtful that one can obtain at present reliable information about the nuclear equation of state from subthreshold antiproton production.

The reduction of the in-medium nucleon and antiproton masses leads to an enhancement of primordial antiproton production as a result of the lower production threshold. To see this explicitly, we carry out three calculations for the antiproton production probability in ${ }^{28} \mathrm{Si}+{ }^{28} \mathrm{Si}$ collision at $2.1 \mathrm{GeV} /$ nucleon and $b=0 \mathrm{fm}$. The first calculation is done in the usual nonrelativistic VUU model, using a soft Skyrme parametrization for the equation of state $(K=200 \mathrm{MeV})[4,5]$. In this case baryon masses do not change with density as the single-particle potential is momentum independent. Bare nucleon and antiproton masses are thus used in Eqs. (12)-(14). The result is shown in Fig. 10 by the dotted curve. The other two calculations are done in the RVUU model. In one calculation, the bare antiproton mass is used; i.e., only nucleon mass drops with density. The threshold in this case is thus $3 m^{*}+m$. The result of this calculation is shown in Fig. 10 by the dashed curve. The antiproton production probability in this case (dashed curve) is enhanced by about a factor of 7 over the result with the bare nucleon mass (dotted curve) (from $3.4 \times 10^{-7}$ to $2.4 \times 10^{-6}$ ). In the final calculation, both effective nucleon and antiproton masses are used and the threshold is therefore $4 \mathrm{~m}^{*}$. The result is shown in Fig. 10 by the solid curve. It is seen that the antiproton production probability is further enhanced by about a factor of 3 as compared to the second case (from $2.4 \times 10^{-6}$ to $7.9 \times 10^{-6}$ ). Overall there is

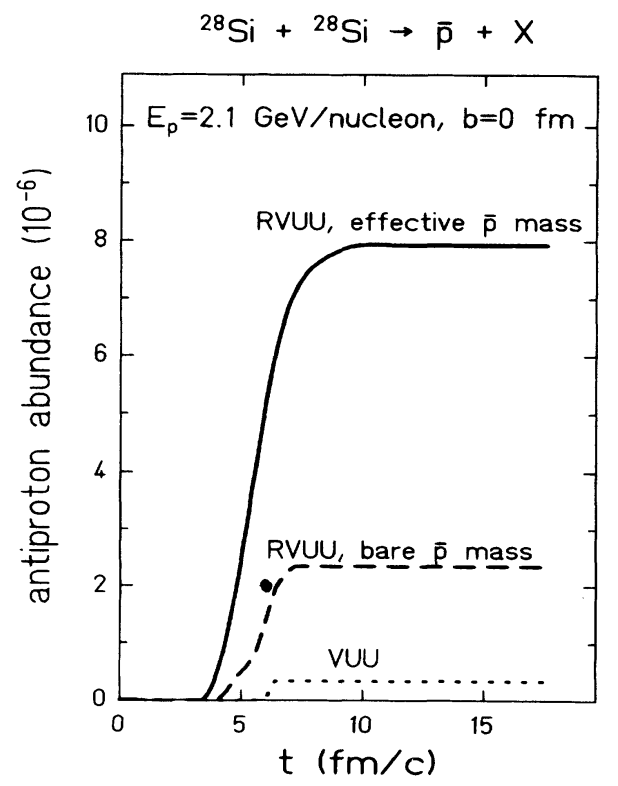

FIG. 10. The primordial antiproton production probability obtained with the VUU model (dotted curve), the RVUU model with the bare antiproton mass (dashed curve), and the RVUU model with the effective antiproton as well as nucleon masses (solid curve). about a factor of 20 enhancement of the antiproton production cross section due to the reduction of the baryon mass in the medium. We note that, in these calculations we have always assumed that the vector potentials in the intial and final states of the reaction $B B \rightarrow N N p \bar{p}$ are the same.

The impact parameter dependence of the antiproton abundance is shown in Fig. 11 for ${ }^{28} \mathrm{Si}+{ }^{28} \mathrm{Si}$ at 2.1 $\mathrm{GeV} /$ nucleon. The dashed and solid curves give the primordial and final (with annihilation and multiplied by a factor of 10) antiprotons, respectively. It is seen that, with increasing impact parameter, the abundance of primordial antiprotons decreases faster than that of final antiprotons. This is reasonable since the annihilation effect is more significant for central collisions than for peripheral ones.

So far we have discussed mainly the antiproton abundance and production probability. It is clear that the propagation of antiproton in the medium [Eqs. (15) and (16)] and the elastic rescattering of antiprotons by baryons do not affect these integrated quantities. These medium effects modify, however, considerably the final antiproton spectrum. In Fig. 12 we show the effect of antiproton propagation in the mean-field potential on its momentum spectrum at $\theta_{\text {lab }}=0^{\circ}$ and $25^{\circ}$ in ${ }^{28} \mathrm{Si}+{ }^{28} \mathrm{Si}$ collision at $2.1 \mathrm{GeV} /$ nucleon and $b=0 \mathrm{fm}$. The solid and dashed curves correspond to the results with and without antiproton propagation in the mean-field potential. Because of the mean-field potential, the antiproton momentum spectrum becomes steeper and more antiprotons with intermediate momenta are produced. A similar effect was found in Ref. [32] for the antiproton transverse momentum spectrum in heavy-ion collisions at the AGS energies, and it can explain qualitatively the difference between the observed proton and antiproton apparent temperatures.

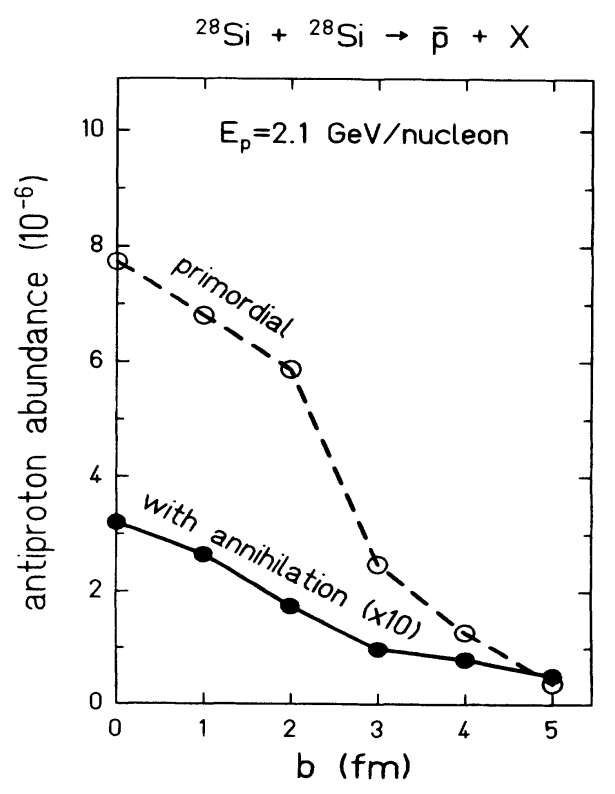

FIG. 11. The impact parameter dependence of the primordial and final (with antiproton annihilation and multiplied by a factor of 10) antiproton abundance. 


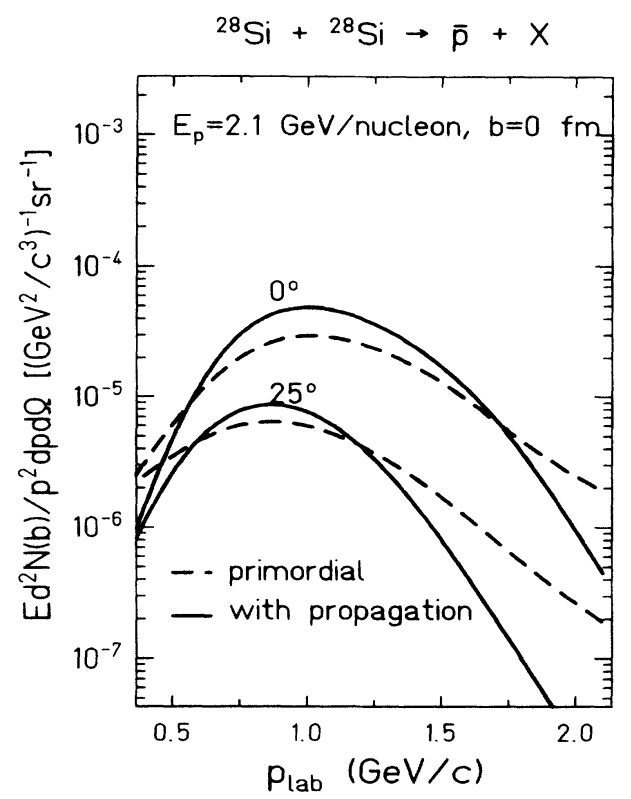

FIG. 12. The antiproton differential momentum distribution at $\theta_{\text {lab }}=0^{\circ}$ and $25^{\circ}$ in ${ }^{28} \mathrm{Si}+{ }^{28} \mathrm{Si}$ collision at 2.1 $\mathrm{GeV} /$ nucleon and $b=0 \mathrm{fm}$. The solid and dashed curves are the results with and without antiproton propagation in the mean-field potential, respectively.

In Fig. 13 we show the effect of antiproton elastic rescattering by baryons on its momentum spectrum. The same collision system as in Fig. 12 is considered here. The solid and dashed curves correspond, respectively, to the results with and without rescattering. On the average, each antiproton undergoes about eight rescatterings (note that in this calculation neither the antiproton propagation in the mean-field potential nor its annihilation is included). With elastic rescattering, the antipro-

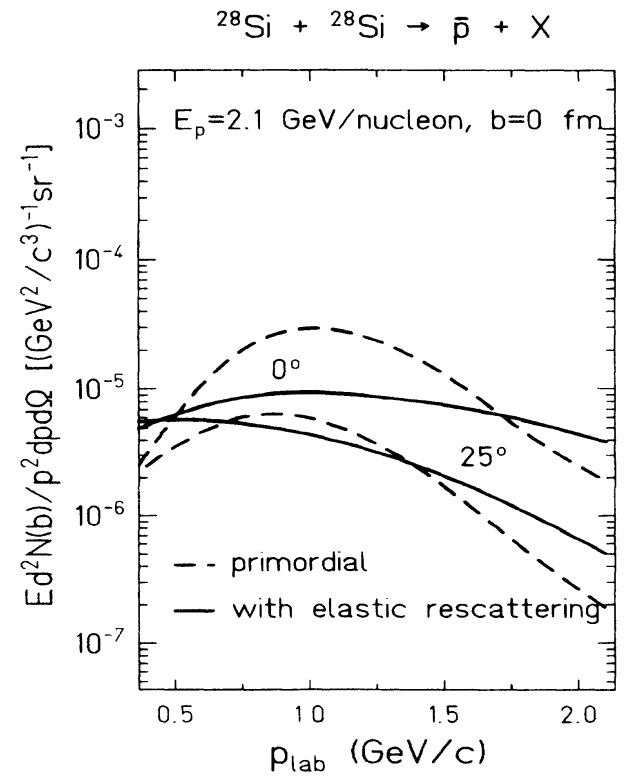

FIG. 13. Same as Fig. 12; the solid and dashed curves are the results with and without elastic rescattering, respectively. ton momentum spectrum becomes flatter. This effect has also been observed in the kaon momentum spectrum from nucleus-nucleus collisions at subthreshold energies [39].

The effect of antiproton annihilation on its momentum spectrum is shown in Fig. 14. In this calculation, both antiproton propagation in the mean-field potential and elastic rescattering are turned off. The solid and dashed curves represent the results with and without annihilation, respectively. We see that the annihilation plays a decisive role in the final antiproton momentum spectrum. With annihilation, the antiproton multiplicity is reduced by almost two orders of magnitude at low momenta and more than one order of magnitude at high momenta. The annihilation is stronger for low-momentum antiprotons as the annihilation cross section increases with decreasing momentum (see Fig. 5). Also, the low-momentum antiprotons stay longer in dense medium and thus have a larger chance to be annihilated. We note that in our calculation the free-space antiproton annihilation cross section [36] has been used. The in-medium annihilation cross section is unknown at present and may be different from the free one. Knowledges on antiproton annihilation in dense matter will be very useful in future studies.

Finally, we compare in Fig. 15 our theoretical prediction for the antiproton differential cross section in ${ }^{28} \mathrm{Si}+{ }^{28} \mathrm{Si}$ collision at $2.1 \mathrm{GeV} /$ nucleon with the experimental data from Ref. [8]. The dashed curve gives the cross section of the primordial antiprotons, while the solid curve represents the final result that takes into account antiproton propagation in the mean-field potential, elastic rescattering, and annihilation. First of all, we note that our theoretical prediction based on a consistent treatment of antiproton production, propagation, rescattering, and annihilation within the RVUU model fits reasonably well the experimental data. If the bare nucleon and antiproton masses are used, i.e., the calculation with

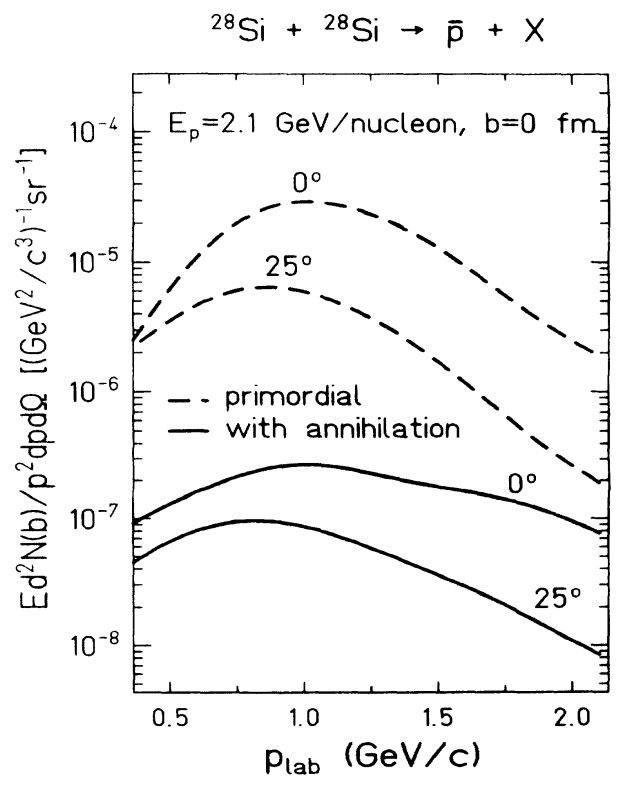

FIG. 14. Same as Fig. 12; the solid and dashed curves are the results with and without annihilation, respectively. 


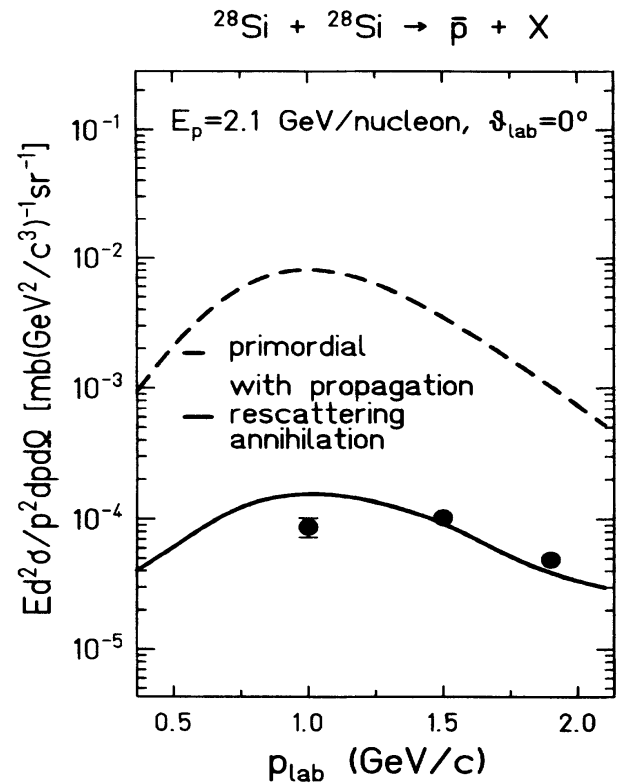

FIG. 15. The antiproton momentum spectrum at $\theta_{\text {lab }}=0^{\circ}$ in ${ }^{28} \mathrm{Si}+{ }^{28} \mathrm{Si}$ at $2.1 \mathrm{GeV} /$ nucleon. The dashed curve is for the primordial antiprotons, while the solid curve is the final result obtained with antiproton propagation, elastic rescattering, and annihilation. The experimental data are taken from Ref. [8].

the nonrelativistic VUU model as in Ref. [19], the experimental data will be underestimated by about a factor of 20, similar to that shown in Fig. 10 for the total production probability. In Ref. [19] the experimental data were underestimated by only about a factor of 5 , since, as we already pointed out early, in their schematic treatment of annihilation, the annihilation probability was also underestimated by about a factor of 3 . Furthermore, if the effective nucleon mass is used but the antiproton mass is assumed to be unaffected by the medium, the experimental data will be underestimated by about a factor of 3 (see discussion about the production probability in Fig. 10). On the other hand, the experimental value at $p_{\text {lab }}=1.0 \mathrm{GeV} / c$ is slightly overestimated by our calculation. This may indicate possible modifications of antiproton momentum distribution from the baryon-baryon interaction and its annihilation in dense matter.

\section{SUMMARY}

In summary, we have generalized the relativistic transport model to include the antiproton degree of freedom, and treated consistently the antiproton production, propagation, elastic rescattering, and annihilation in the hadronic matter. The antiproton self-energies have been obtained, in the mean-field approximation, from the $G$ parity transformation of the nucleon self-energies. Our main conclusions can be summarized as follows.

(1) As pointed out in Refs. $[15,19,40]$, the delta degree of freedom is very important in accounting for the large antiproton yield in nucleus-nucleus collisions at subthreshold energies. Without deltas, the antiproton yield will be greatly underestimated. This is one of the reasons for the small antiproton production cross section obtained in the calculation of Ref. [17]. Higher resonances have not been included in the present study, and their effects on subthreshold antiproton production needs to be investigated.

(2) An explicit treatment of antiproton propagation and elastic rescattering by baryons is necessary as they modify significantly the antiproton momentum spectrum in nucleus-nucleus collisions. These medium effects have been investigated in Ref. [41] for the calculation of antiproton production at AGS energies in the framework of relativistic quantum molecular dynamics (RQMD).

(3) Antiproton annihilation plays a decisive role on the final antiproton yield and momentum spectrum. It needs to be included explicitly in the transport model. In our study, the antiproton survival rate is only about $4 \%$, and is much smaller than that in Ref. [40]. This discrepancy needs to be understood in future studies.

(4) In our study, it is necessary to include medium effects on nucleons and antiprotons in order to account for the experimental antiproton yield in nucleus-nucleus collisions. With the in-medium effective nucleon and antiproton masses, the antiproton yield is enhanced due to the reduction of the production threshold. In particular, our assumption that the antiproton potential in dense matter is given by the $G$-parity transformation of the nucleon potential leads to a reasonable agreement with the experimental data. However, one needs to be aware of the uncertainty in the elementary antiproton production cross section close to the threshold. Also, the contributions from the higher resonances may be non-negligible. All these may affect our results, and more work is thus required in the future.

In conclusion, our results are quite encouraging. Since collisions with nuclei heavier than $\mathrm{Si}$ can lead to a larger volume of high density matter in the initial compressional stage of the collision, medium effects are expected to be more appreciable in such reactions. It is thus of interest to continue in the future similar studies of antiproton production for collisions with heavier nuclei.

This work was supported in part by the National Science Foundation under Grant No. PHY-9212209 and the Welch Foundation under Grant No. A-1110.
[1] J. Aichelin and C. M. Ko, Phys. Rev. Lett. 55, 2661 (1985).

[2] W. Cassing, W. Metag, U. Mosel, and K. Niita, Phys. Rep. 188, 363 (1990).
[3] U. Mosel, Annu. Rev. Nucl. Part. Sci. 41, 29 (1991).

[4] J. Aichelin, Phys. Rep. 202, 235 (1991).

[5] G. Q. Li, A. Faessler, and S. W. Huang, Prog. Part. Nucl. Phys. 31, 159 (1993). 
[6] J. Carroll, Nucl. Phys. A488, 203c (1988).

[7] E. Grosse, Prog. Part. Nucl. Phys. 30, 89 (1993).

[8] J. Carroll, S. Carlson, J. Gordon, T. Hallman, G. Igo, P. Kirk, G. F. Krebs, P. Lindstrom, M. A. McMahan, V. Perez-Mendez, A. Shor, S. Trentalange, and Z. F. Wang, Phys. Rev. Lett. 62, 1829 (1989); A. Shor, E. F. Barasch, J. B. Carroll, T. Hallman, G. Igo, G. Kalnins, P. Kirk, G. F. Krebs, P. Lindstrom, M. A. McMahan, V. PerezMendez, S. Trantalange, F. J. Urban, and Z. F. Wang, ibid. 63, 2192 (1989).

[9] A. A. Baldin et al., Pis'ma Zh. Eksp. Teor. Fiz. 48, 127 (1988) [JETP Lett. 48, 137 (1988)]; A. A. Baldin, Yu. K. Gavrilov, F. F. Guber, A. B. Kurepin, V. S. Pantuev, M. A. Prokhvatilov, V. I. Razin, A. I. Reshetin, and S. N. Filippov, Nucl. Phys. A519, 407c (1990).

[10] O. Chamberlain, W. W. Chupp, G. Goldhaber, E. Segre, C. Wiegand, E. Amaldi, G. Baroni, C. Castagnoli, C. Franzinetti, and A. Manfredini, Nuovo Cimento, 3, 447 (1956).

[11] T. Elioff, L. Agnew, O. Chamberlain, H. M. Steiner, C. Wiegand, and T. Ypsilantis, Phys. Rev. 128, 869 (1962).

[12] D. E. Dorfan, J. Eades, L. M. Lederman, W. Lee, C. C. Ting, P. Piroue, S. Smith, J. L. Brown, J. A. Kadyk, and G. H. Trilling, Phys. Rev. Lett. 14, 995 (1965).

[13] J. Chiba, D. Ashery, H. Ito, K. Kimura, Yu. T. Kiselev, S. Koda, K. Miyano, T. Murakami, T. Nagae, Y. Nakai, M. Nomachi, S. Sawada, M. Sekimoto, T. Suzuki, K. H. Tanaka, M. K. Vlasov, and Y. Yoshimura, Nucl. Phys. A553, 771c (1993).

[14] A. Schröter, E. Berdermann, H. Geissel, A. Gillitzer, J. Homolka, P. Kienle, W. Koenig, B. Povh, F. Schumacher, and H. Stöcker, Nucl. Phys. A553, 775c (1993).

[15] P. Koch and C. B. Dover, Phys. Rev. C 40, 145 (1989).

16] C. M. Ko and X. Ge, Phys. Lett. B 205, 195 (1988); C. M. Ko and L. H. Xia, Phys. Rev. C 40, R1118 (1989).

[17] A. Shor, V. Perez-Mendez, and K. Ganezer, Nucl. Phys. A514, 717 (1990).

[18] P. Danielewicz, Phys. Rev. C 42, 1564 (1990).

[19] G. Batko, W. Cassing, U. Mosel, K. Niita, and Gy. Wolf, Phys. Lett. B 256, 331 (1991).

[20] S. W. Huang, G. Q. Li, T. Maruyama, and A. Faessler, Nucl. Phys. A547, 653 (1992).

[21] J. Schaffner, I. N. Mishustin, L. M. Satarov, H. Stöcker, and W. Greiner, Z. Phys. A 341, 47 (1991).
[22] W. Cassing, A. Lang, S. Teis, and K. Weber, Nucl. Phys. A545, 123c (1992).

[23] B. D. Serot and J. D. Walecka, Adv. Nucl. Phys. 16, 1 (1986).

[24] J. Boguta and A. R. Bodmer, Nucl. Phys. A292, 413 (1977).

[25] C. M. Ko, Q. Li, and R. Wang, Phys. Rev. Lett. 59, 1084 (1987); Q. Li and C. M. Ko, Mod. Phys. Lett. A 3, 465 (1988); C. M. Ko and Q. Li, Phys. Rev. C 37, 2270 (1988); Q. Li, J. Q. Wu, and C. M. Ko, ibid. 39, 84 (1989); C. M. Ko, Nucl. Phys. A495, 321c (1989).

[26] W. Botermans and R. Malfliet, Phys. Rep. 198, 115 (1990).

[27] S. J. Wang, B. A. Li, and J. Randrup, Ann. Phys. (N.Y.) 209, 251 (1991).

[28] C. Y. Wong, Phys. Rev. C 25, 1461 (1982).

[29] J. Cugnon, D. Kinet, and J. Vandermeullen, Nucl. Phys. A379, 553 (1982).

[30] G. F. Bertsch and S. Das Gupta, Phys. Rep. 160, 189 (1988).

[31] P. Danielewicz and G. F. Bertsch, Nucl. Phys. A533, 712 (1991).

[32] V. Koch, G. E. Brown, and C. M. Ko, Phys. Lett. B 265, 29 (1991).

[33] T. K. Gaisser and F. Halzen, Phys. Rev. D 11, 3157 (1975).

[34] C. S. Shen and G. B. Berkey, Phys. Rev. 171, 1344 (1968).

[35] M. Antinucci, A. Bertin, P. Capiluppi, M. D'AgostinoBruno, A. M. Rossi, G. Vannini, G. Giacomelli, and A. Bussiere, Lett. Nuovo Cimento 6, 121 (1973).

[36] J. Cugnon and J. Vandermeulen, Ann. Phys. (Paris) 14, 49 (1989).

[37] V. Flaminio et al., Report No. CERN-HERA 84-01, 1984.

[38] S. W. Huang, A. Faessler, G. Q. Li, R. K. Puri, E. Lehmann, D. T. Khoa, and M. A. Matin, Phys. Lett. B 298, 41 (1993).

[39] X. S. Fang, C. M. Ko, and Y. M. Zheng, Nucl. Phys. A556, 499(1993); X. S. Fang, C. M. Ko, G. Q. Li, and Y. M. Zheng, Phys. Rev. C (in press).

[40] C. Spieles, A. Jahns, H. Stöcker, and W. Greiner, Mod. Phys. Lett. A 27, 2547 (1993).

[41] A. Jahns, H. Stöcker, W. Greiner, and H. Sorge, Phys. Rev. Lett. 68, 2895 (1992). 